\title{
Mesure des plus-values en capital et du statut économique des ménages agricoles
}

Measuring the capital gains and economic status of farm households

\section{Berkeley Hill}

Traducteur : Catherine Moreddu

\section{(2) OpenEdition Journals}

\section{Édition électronique}

URL : http://journals.openedition.org/economierurale/127

DOI : 10.4000/economierurale. 127

ISSN : 2105-2581

\section{Éditeur}

Société Française d'Économie Rurale (SFER)

\section{Édition imprimée}

Date de publication : 30 décembre 2005

Pagination : 63-74

ISSN : 0013-0559

Référence électronique

Berkeley Hill, « Mesure des plus-values en capital et du statut économique des ménages agricoles », Économie rurale [En ligne], 289-290 | Septembre-décembre 2005, mis en ligne le 02 mars 2009, consulté le 01 mai 2019. URL : http://journals.openedition.org/economierurale/127 ; DOI : 10.4000/ economierurale.127

Ce document a été généré automatiquement le 1 mai 2019.

(c) Tous droits réservés 


\title{
Mesure des plus-values en capital et du statut économique des ménages agricoles
}

\author{
Measuring the capital gains and economic status of farm households
}

Berkeley Hill

Traduction : Catherine Moreddu

\section{NOTE DE L'ÉDITEUR}

Traduction de Catherine Moreddu, Ocde

\section{Introduction}

1 Par bien des aspects, le patrimoine a une grande influence sur l'évolution de la situation économique des familles agricoles. Non seulement le patrimoine génère différentes formes de flux de revenu, mais il apporte la sécurité, la liberté de manœuvre et le pouvoir économique et politique (Hill, 2000). L'accumulation du patrimoine (plutôt que celle du simple revenu) et son transfert entre générations ont un rôle déterminant dans les décisions des exploitants agricoles. Le patrimoine est un facteur sous-jacent mais crucial qui doit être pris en compte lorsqu'on essaie d'expliquer le comportement des ménages agricoles.

2 L'information sur le patrimoine peut contribuer à la formulation des problèmes que les politiques agricoles essaient de traiter. Il ajoute, en particulier, une autre dimension à la notion de niveau de vie adéquatqui constitue un objectif fondamental de la politique agricole commune (PAC) de l'Union européenne. Au sein de la société dans son ensemble, le patrimoine semble présenter une répartition beaucoup plus inégale que celle du revenu et il a une grande influence sur le degré général d'inégalité (Atkinson, 1980). En tant que 
groupe, il semble que dans de nombreux pays, les ménages agricoles possèdent un patrimoine plus important que la société dans son ensemble; en France, la valeur moyenne de leurs actifs représente environ le double de celle des autres indépendants et des salariés (Jégouzo et al., 1998). Aux États-Unis en 1995, un rapport similaire existait entre les fonds propres (actifs moins dettes) des ménages agricoles et ceux de la moyenne des ménages américains (Sommer et al., 1998). Outre son importance pour la conception des politiques, l'information sur le patrimoine peut apporter des éclaircissements sur les effets des interventions, car elles ont souvent une incidence sur la valeur des actifs.

En réalité, le patrimoine n'est pas suffisamment étudié et pris en compte dans les statistiques portant sur l'agriculture et les exploitants agricoles. Les Comptes économiques de l'agriculture (CEA) officiels de l'Union européenne ne comprennent pas le bilan des actifs et des dettes; les variations de richesse de l'agriculture ne sont décrites que par des indicateurs des rémunérations de l'activité agricole courante. Le Réseau d'information comptable agricole (RICA) donne une image partielle des fonds propres, mais il ne couvre que les actifs et les emprunts dits agricoles. Là encore, l'attention se porte sur des indicateurs du revenu courant (tiré uniquement de la production agricole) et on accorde peu d'attention à ce que les fonds propres deviennent, compte tenu des concepts sur lesquels se fondent les CEA et le RICA. Dans les deux cas, il s'agit strictement de comptes d'activités plutôt que de comptes portant sur des unités institutionnelles (ménages/exploitations et entreprises). Seules les unités institutionnelles sont capables de posséder des actifs et d'encourir des dettes, et donc de posséder des fonds propres. En particulier, il est à la fois discutable au plan théorique (du fait de la nature fongible des prêts) et difficile au plan pratique d'essayer de séparer les dettes agricoles.

Dans un contexte de politique agricole, cet article porte principalement sur deux aspects $\mathrm{du}$ patrimoine qui, récemment, n'ont pas beaucoup retenu l'attention des économistes agricoles. Il s'agit :

1. Des plus-values en capital que les actifs peuvent apporter à leurs propriétaires.

2. De l'importante contribution du patrimoine au bien-être économique des agriculteurs.

\section{Plus-values en capital et revenu}

5 Selon la notion hicksienne de revenu (Hicks, 1946), les plus-values réelles (c'est-à-dire celles qui vont au-delà des changements de valeur des actifs dus à l'inflation) font partie du revenu personnel du propriétaire des actifs. Les statistiques qui ne portent que sur les revenus courants ne sont donc capables de représenter qu'une partie de la situation en matière de revenu. Cependant, les plus-values (et les moins-values qui peuvent être considérées comme des gains négatifs) diffèrent du revenu courant par bien des aspects (en termes de liquidité, de risque, de mesurabilité, etc.). Il est donc probablement inapproprié de faire la somme des deux éléments. Les plus-values, en particulier celles concernant le foncier agricole, tendent à bénéficier d'un traitement fiscal plus avantageux que le revenu courant, ce qui encourage les entrepreneurs à se tourner vers cette forme de rémunération (Andersen et al., 2002). Elles ne correspondent pas facilement aux conventions des CEA agrégés (même si elles ont leur place dans la méthodologie des comptes nationaux). Elles sont pour la plupart exclues du cadre de la comptabilité microéconomique agricole de l'Union européenne. Il faudrait cependant les prendre en compte. 
6 La littérature établit clairement que le soutien à la production agricole est directement capitalisé dans la valeur des actifs, les propriétaires fonciers étant les principaux bénéficiaires car la terre est l'actif dont l'offre est la moins élastique (OCDE, 1998). Ces propriétaires fonciers peuvent ou non être des exploitants agricoles. La valeur des terres est, en partie, une représentation de la valeur marginale future escomptée du produit de l'utilisation de ce facteur dans l'agriculture ou, peut-être, dans des activités alternatives (comme la construction immobilière ou les terrains de golf). Lorsque les attentes changent, il est probable que les plus (ou moins)-values en capital varient aussi. Par exemple, une réforme de la politique agricole qui diminue les profits escomptés entraînerait une baisse des prix de marché de la terre, tandis qu'une modification des règles de planification permettant la construction immobilière conduirait probablement à leur hausse. La cause des plus (ou moins)-values n'a pas d'importance pour le calcul $a$ posteriori du revenu personnel au sens hicksien du terme car les flux de revenu courant et la hausse de la valeur réelle des actifs sur la même période donnent la possibilité de consommer sans réduire la valeur nette des actifs.

7 Étant donné les diverses manières dont la rémunération de l'agriculture peut être capturée et dont les différents mécanismes de soutien affectent les revenus et la valeur des actifs, les analystes des politiques se sont intéressés à la répartition des avantages du soutien (OCDE, 2003).

8 Au niveau du ménage/entreprise agricole, les plus et moins-values en capital ont des répercussions directes sur toute une gamme de facteurs liés au comportement. Elles influencent les décisions en matière de production et d'investissement au sein de l'entreprise agricole et les niveaux de consommation des exploitants. Elles affectent l'épargne, les agriculteurs ayant une plus forte tendance à économiser une partie de leurs plus-values que leur revenu courant (Bhatia, 1972). Un effet important des plus-values est qu'elles modifient sans doute l'attitude des propriétaires-exploitants face au risque, ce qui accroît le patrimoine personnel. Selon Hearn (1977) ${ }^{1}$, les agriculteurs qui ont engrangé des plus-values pourraient investir et s'engager dans des activités plus risquées et leurs activités agricoles seraient donc différentes de celles des agriculteurs qui n'ont pas fait de plus-values. Il existe diverses manières de transformer les plus-values en moyens de dépenses, y compris par accroissement de la capacité d'emprunt du fait d'une amélioration du bilan et par diminution des prélèvements sur le revenu courant pour préparer la pension de retraite. Au Royaume-Uni, il y a une grande diversité de vue parmi les agriculteurs concernant leur perception de l'impact des plus-values sur leur capacité d'emprunt, les petites exploitations soulignant plus fortement l'avantage que ces plusvalues procurent en terme d'accès au crédit que les exploitations moyennes ou plus grandes dont les revenus sont plus importants (Hearn, op. cit.). Les plus-values influent aussi sur la décision des agriculteurs de rester dans le secteur ou de le quitter; les anticipations concernant la hausse des prix des terres et les avantages fiscaux dont bénéficient les plus-values, en particulier en terme de retraite, ont été considérés comme un frein déterminant au changement structurel qui mènerait à une taille d'exploitation plus conforme aux conditions techniques et économiques actuelles (Hearn, op. cit.; Perry et al., 1986). 


\section{Qui calcule les plus-values en capital des ménages agricoles?}

9 Dans les années 1970, une hausse continue des prix des terres et de la valeur des actifs a été enregistrée dans de nombreux pays industrialisés, ce qui a éveillé l'intérêt dans la littérature universitaire sur la taille relative des plus-values en capital et des revenus courants. Le développement des statistiques économiques n'a pas suivi. Dans l'Union européenne (et au Royaume-Uni), il n'existe pas de statistiques officielles sur les plus ou moins values agrégées de l'industrie agricole. De telles estimations existent cependant dans plusieurs pays. Aux États-Unis par exemple, des chiffres sur les gains et pertes (nominaux et réels) en capital ont été calculés par le Département de l'agriculture (USDA) à partir de 1960 et à un moment, ils étaient publiés dans la revue annuelle du secteur agricole «Farm Sector Review». Avant cette date, il faut consulter des sources non officielles. Les estimations de Melichar (1979) s'accompagnaient du commentaire suivant : sur les deux décennies précédant 1972, les plus-values réelles se montaient en moyenne à un tiers du revenu agricole net (" over the two decades preceding 1972 real capital gains averaged about one-third of net farm income ») $)^{2}$. Tout au long de ces années, des gains ont été enregistrés en termes réels à un taux supérieur au revenu agricole net agrégé. Selon diverses estimations, les plus-values auraient commencé à prendre de l'importance dans l'agriculture américaine aux alentours de 1968 ; à partir de là, l'immobilier a commencé à procurer des rendements supérieurs et plus réguliers qu'au début des années de l'immédiate après-guerre, et ces gains atteignaient des niveaux qui n'avaient été enregistrés auparavant qu'à titre exceptionnel (1950, 1956 et 1958). La plupart des gains provenaient de l'immobilier; sur la période 1947-1968, Bhatia (1970) a estimé que 88 pour cent des plus-values réelles totales avaient cette origine.

La forte baisse des prix de l'immobilier après 1981 a fait diminuer l'intérêt dans la littérature pour cette forme de rémunération de l'agriculture. Il ne semble pas y avoir eu d'écrits importants sur les incidences des moins-values en capital. Aux États-Unis cependant, des pertes ont été enregistrées à partir de la fin des années 1980 et des plusvalues sur l'immobilier ont de nouveau été enregistrées dans les années 1990 (graphique 1). Sur l'ensemble de la période 1960-97, les plus-values réelles sur le foncier et les bâtiments ont dépassé marginalement les pertes (32 millions de dollars des États-Unis en valeur 1990) mais elles ont dépassé les gains sur l'endettement agricole (166 millions de dollars des États-Unis) ; les pertes sur les autres actifs de l'entreprise (y compris les stocks de cultures et les actifs financiers) ont réduit les gains en capital propre à 182 millions de dollars des États-Unis. Il est clair d'après les estimations américaines que les années sur lesquelles la mesure est effectuée peuvent avoir une grande influence sur l'incidence des gains et pertes sur la rémunération totale, et que la terre doit être possédée depuis très longtemps avant qu'apparaisse un gain net. 
Graphique 1. -États-Unis : estimation des plus et moins-values sur l'immobilier et l'endettement agricole (1960-1997)

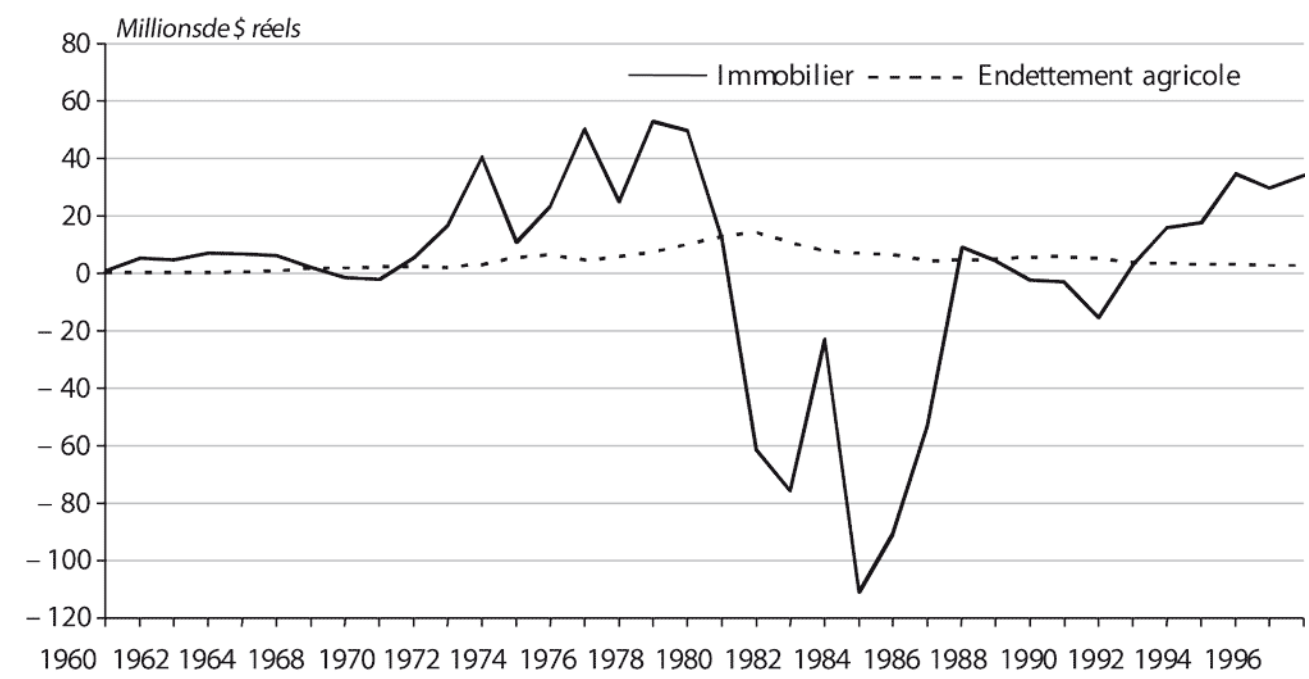

11 Le Canada a aussi estimé les plus-values en capital agricole. Comme aux États-Unis, leur importance par rapport au revenu a augmenté au cours des années 1970 (Brinkman, 1980 ; Chase, 1980).

12 Le Danemark est une exception parmi les quinze pays membres de l'Union européenne dans la mesure où il a publié des estimations des plus et moins-values en capital dans le cadre de son enquête annuelle officielle du secteur agricole. Le poids des dettes dans l'agriculture danoise rend cette prise en compte des gains et pertes sur les actifs financiers particulièrement importante. La Suède a également publié sur une longue période des estimations officielles des plus-values, notamment sur les propriétés agricoles et les dettes.

Ces estimations sont réalisées au niveau agrégé du secteur et ne sont liées que de manière indirecte aux plus et moins-values en capital qui entrent dans la formation des revenus personnels des agriculteurs et de leurs ménages. Parmi les études universitaires portant sur des exploitations agricoles individuelles, on trouve celle de Gardner (1975) qui a estimé les revenus des agriculteurs, y compris les plus-values en capital non réalisées d'une année "normale». Cela a conduit à une réduction substantielle du nombre de familles agricoles situées sous le seuil de pauvreté. En examinant les plus-values en capital en Angleterre à l'aide d'une enquête des exploitations agricoles, Harrison (1975) a trouvé que les plus-values en capital n'étaient pas réparties du tout de façon égalitaire entre agriculteurs mais se concentraient sur les plus grands et les plus anciens propriétaires fonciers. En outre, les agriculteurs n'ont pas réagi de façon identique, même s'il se peut qu'ils aient enregistré des gains identiques au total et dans le temps. Aux États-Unis, on a noté des différences régionales en gains et pertes en capital qui pourraient être reliées aux attentes en matière de revenu résultant des décisions des pouvoirs publics concernant les programmes de soutien des produits de base, ce qui implique que certains types d'exploitations étaient plus touchés que d'autres (Weldon et al., 1993).

Les travaux sur les plus-values en capital se fondent en général sur des données relatives aux actifs agricoles. Dans le capital, il est possible de distinguer les éléments agricoles des éléments non agricoles (même si c'est difficile lorsqu'on considère des proportions, par 
exemple concernant des véhicules), mais des problèmes théoriques et pratiques se posent pour séparer les emprunts agricoles. Bien entendu, si l'exploitant agricole et sa famille possèdent aussi d'autres actifs (actifs non agricoles), les plus et moins values en capital sur ceux-ci auront des incidences sur les décisions relatives à la gestion de l'exploitation et à la consommation. Par exemple, une chute de la valeur des actifs non agricoles aura probablement pour effet de freiner toute décision sur l'utilisation du capital de l'exploitation comportant des risques importants. Il est donc souhaitable, comme dans les bilans comptables, de connaître les gains et pertes au sein de l'unité ménage-exploitation qui pourraient expliquer le cadre plus large de l'entreprise agricole.

Les évolutions actuelles de la politique agricole de l'Union européenne (baisse des prix des produits de base, compensations directes des revenus, paiements pour des services environnementaux, etc.) auront probablement des incidences sur les prix des actifs et affecteront donc la rémunération globale des ménages-exploitations agricoles. Ces effets ne seront ni simples ni uniformes. Aux États-Unis, où le lien entre le soutien et le prix des terres est probablement plus direct, on s'attend à ce que des ajustements importants à la politique agricole interviennent à travers la baisse de la valeur des actifs (Blandford et Boisvert, 2004). Il serait donc utile de mettre en place un système de suivi des plus et moins values en capital.

\section{Statut économique des ménages agricoles}

16 L'ampleur relative du patrimoine détenu par les ménages agricoles aux États-Unis et en France a déjà été mentionnée. Des informations isolées laissent à penser que des situations similaires peuvent se trouver dans la plupart des pays de l'Union européenne et ailleurs. Lorsqu'on passe des agriculteurs en général à ceux dont le faible niveau de vie est particulièrement préoccupant, il est paradoxal que dans de nombreux pays industrialisés, les agriculteurs à faible revenu soient fréquemment détenteurs d'un patrimoine conséquent, dont le montant est en moyenne supérieur à celui des ménages non agricoles. Aux États-Unis en 1986, les actifs agricoles des exploitants agricoles dont les revenus étaient inférieurs au seuil de pauvreté étaient bien supérieurs à la moyenne des fonds propres de l'ensemble des ménages (Ahearn et Lee, 1991). Plus de la moitié de ces ménages agricoles pauvres était constituée de familles dont le chef avait l'agriculture comme principale occupation et dont la principale source de revenu était l'activité agricole. Les actifs agricoles moyens de ces ménages à faible revenu et dépendant de l'agriculture se montaient à 238640 dollars des États-Unis, soit plus de trois fois la moyenne nationale de l'ensemble des ménages.

La combinaison patrimoine élevé/faible revenu, qui est particulièrement caractéristique des agriculteurs âgés, devrait attirer l'attention sur la façon dont le patrimoine est pris en compte dans l'évaluation du statut économique et sur les critères utilisés pour le soutien public aux revenus. Il pourrait suggérer comment le patrimoine peut être utilisé pour contribuer aux dépenses courantes, en évitant le recours aux transferts en provenance d'autres secteurs résultant de la plupart des mécanismes de politique.

Le « statut économique » d'un individu, c'est-à-dire sa consommation potentielle de biens et services, est lié à la fois à son revenu courant et à ses fonds propres. Le patrimoine représente la capacité potentielle à dépenser. Deux individus possédant un même revenu courant mais des montants d'actifs différents auront un potentiel de consommation différent. Pour exprimer le revenu et le patrimoine dans une mesure commune, il est 
courant de calculer la rente annuelle des fonds propres c'est-à-dire le flux de revenu annuel correspondant au montant total. Elle s'ajoute ensuite à la mesure conventionnelle $\mathrm{du}$ revenu pour donner un paramètre du flux total de services économiques à la disposition de l'unité de consommation.

$$
\begin{gathered}
19 \text { Statut économique = } \\
\text { revenu courant + rente annuelle } \\
\text { correspondant à la valeur des fonds propres }
\end{gathered}
$$
ée pour les agriculteurs, mais on y a porté peu d'intérêt. Pour l'année 1966 aux États-Unis, Carlin et Reinsel (1973) ont trouvé que la répartition du bien-être parmi les familles agricoles devenait plus égale lorsque le patrimoine annualisé était ajouté au revenu courant dans une mesure conjointe, et que les disparités entre le secteur agricole et les autres secteurs se réduisaient dans la mesure où la situation des familles agricoles s'améliorait. Des résultats semblables ont été rapportés pour des familles agricoles du Dakota du nord en 1970 (Gardner, 1972). En Australie, selon des recherches sur la pauvreté des agriculteurs dans trois régions de l'État de Victoria (Vincent et al., 1975), le nombre des familles situées au-dessous d'un seuil de pauvreté portant sur le revenu serait nettement inférieur si le patrimoine annualisé était aussi pris en compte. Au Canada, la méthode appliquée à un niveau agrégé (celui du secteur des ménages agricoles) (Chase, 1980 ; Chase et Lerohl, 1981) sur la base d'une gamme de taux d'intérêt fondée sur les taux de crédit agricole des secteurs privés et publics, et sur plusieurs niveaux d'espérance de vie, a révélé qu'en 1967, la rente annuelle moyenne agricole ajoutait environ 47 pour cent au bien-être économique total (revenu agricole et non agricole) et qu'en 1977, elle ajoutait 57 pour cent. Ces estimations correspondent à une période où des plus-values étaient enregistrées et les conclusions sont que sur la période 1967-77, les fonds propres représentaient une part croissante du bien-être économique total (statut). Pour la France, un calcul illustratif (Jégouzo et al., 1998) ${ }^{3}$ a montré qu'annualiser les fonds propres moyens des ménages agricoles considérés comme pauvres (revenu total par unité de consommation inférieur à 30000 francs) ajoutait 17300 francs par personne à leur revenu (en supposant une espérance de vie de 30 ans et un taux d'intérêt de 4 pour cent). 

variables demandent un peu plus d'attention. Le revenu courant devrait comprendre toutes les sources de revenu et pas seulement le revenu agricole. De plus, les impôts et autres dépenses indispensables devraient être déduits. Le revenu courant correspond au revenu disponible net (RDN), bien qu'on puisse préférer un indicateur brut (RDB), car les dépenses en éléments du capital peuvent être différées à court terme, ou bien utiliser le revenu monétaire. L'objectif est d'établir un concept de revenu qui reflète au mieux le contrôle potentiel sur les biens et services de consommation. calcul) est sans doute le moins contentieux, même si un peu d'arbitraire est inévitable. La question cruciale pour fixer le taux est de considérer le manque à gagner lorsque le patrimoine est détenu sous forme d'actifs agricoles ou en d'autres termes, le taux qui pourrait être obtenu si les fonds étaient investis ailleurs. Bien que, en simplifiant, les rendements des placements publics à faible risque ou des comptes en banque pourraient constituer une référence pratique, une approche plus complexe pourrait consister à fixer des taux individuels pour chaque propriétaire sur la base de facteurs tels que l'attitude face au risque. $\mathrm{Au}$ niveau individuel, l'espérance de vie (deuxième élément) serait évidemment celle du propriétaire et on peut utiliser des tables fondées sur l'information détenue par les assureurs. Pour des couples d'exploitants agricoles, il n'est pas inenvisageable d'utiliser celle de la personne supposée vivre le plus longtemps. Même lorsque le patrimoine est formellement détenu en commun, on pourrait envisager, en pratique, d'annualiser sur la durée du membre du ménage ayant la plus longue espérance de vie. C'est particulièrement justifié lorsque le revenu courant est mesuré pour un couple du ménage, ce qui serait le cas lorsque le revenu disponible est estimét. Quelque soit l'approche employée, le calcul de la rente annuelle (sous sa forme la plus simple) fait l'hypothèse que le capital est complètement consommé sur la durée de vie escomptée. Aucune part n'est réservée aux transferts entre générations. L'argument souvent entendu est que le patrimoine agricole est en quelque sorte "détenu en trust" pour les générations à venir et donc que ses propriétaires actuels ne sont pas libres de le vendre et de consommer sa valeur. Les experts non agricoles rejetteront probablement cet argument, le considérant comme une plaidoirie de recherche de rente.

Enfin, la rente annuelle, le troisième élément du calcul, peut cependant être ajustée de plusieurs manières pour traduire ces questions si l'on accepte l'argument "en trust " dans la mesure où une partie du capital devrait être mis de côté. En outre, Chase et Lerohl font remarquer que le revenu annuel courant doit être indépendant des fonds propres pour que les deux flux s'ajoutent. C'est un problème, par exemple avec la propriété agricole qui est à la fois une forme de patrimoine personnel et d'actifs d'entreprise. Les agriculteurs ne peuvent en pratique percevoir une rente annuelle fondée sur la totalité de leurs fonds propres sans perdre la source de leur revenu courant. Ce n'est pas le cas lorsque d'autres formes de propriété personnelle sont considérées, à l'exception de la résidence principale, pour laquelle le revenu en nature que représente le logement disparaîtrait en cas de vente. La solution de Chase et Lerohl était de supprimer la rémunération des terres et du capital de leurs estimations du revenu de l'activité agricole. Une alternative consiste à garder le revenu courant tel quel mais à envisager des manières de créer une indépendance entre les deux éléments qui entraîne un ajustement du montant de la rente annuelle. 
27 Il existe deux manières principales d'exprimer les fonds propres en équivalent revenu tout en conservant les actifs agricoles qui génèrent le revenu courant.

- -La première consiste en un accord contractuel de vente à terme par lequel l'agriculteur hypothèque sa propriété en échange d'une rente annuelle fondée sur la valeur nette de la propriété, mais le créancier ne devient formellement propriétaire qu'à la mort de l'agriculteur. Dans ce type d'accord, la valeur des actifs pour le créancier, et donc la somme sur laquelle la rente annuelle est fondée, n'est pas le prix de vente courant mais le prix à la mort de l'agriculteur actualisé au présent. Il existe plusieurs approches pour estimer la valeur actualisée des terres. Dans le cas de l'Australie, Sexton et Duffus (1977) ont supposé que la valeur présente des terres n'était déterminée que par les flux de revenu agricole futurs. Ainsi, la valeur présente de la future valeur des terres a pu être obtenue en déduisant les flux de revenu actualisés reçus pendant la durée de vie escomptée de l'agriculteur. Lorsque des facteurs autres que les profits agricoles déterminent le prix des terres, comme c'est le cas au Royaume-Uni, il pourrait être préférable d'évaluer le probable prix futur des terres et le taux d'intérêt d'opportunités d'investissement alternatifs pour actualiser ces valeurs au temps présent. Dans l'exemple australien, la rente annuelle était ajoutée à la somme des revenus courants de tous les membres de la famille, y compris les gains d'activités non agricoles, les revenus d'investissements (d'autres propriétés qui n'entraient pas dans la rente annuelle) et de sources de revenu non imposables (allocations familiales, etc.). Un taux d'intérêt arbitraire de 6 pour cent a été employé. L'incidence de l'inclusion des accords contractuels à terme de rente annuelle sur le nombre d'agriculteurs australiens considérés comme pauvres a été nette. Dans le secteur laitier, la part des familles aux revenus inférieurs à 4000 dollars en 1974 a baissé de 19 à 5 pour cent. Dans le secteur des raisins secs, la baisse a été de 25 à 14 pour cent, et dans le secteur des pommes et des poires de 29 à 9 pour cent. Avec une classification selon l'âge de l'agriculteur, une réduction de la part des agriculteurs à statut insuffisant a été observée dans tous les groupes mais les plus fortes réductions ont chaque fois été enregistrées dans les familles dont le chef avait dépassé l'âge de la retraite (60 ans et plus). Dans le secteur laitier, 30 pour cent des familles dont le chef avait plus de 60 ans se situaient sous le seuil de pauvreté arbitraire de 4000 dollars sur la base du revenu courant mais ils n'étaient plus que 1 pour cent après inclusion de la rente annuelle. Si un dispositif pratique permettant d'échanger des fonds propres sous forme de terre était mis en œuvre, il aurait clairement une grande incidence sur le statut économique des agriculteurs plus âgés.

- -L'autre alternative permettant de conserver la terre dans les pays qui ont un système de tenure, serait d'évaluer la valeur des terres à partir des accords de vente-location dans lesquels l'agriculteur reste l'exploitant de la ferme mais paie un loyer au nouveau propriétaire. Une estimation pour le Royaume-Uni (Hill, 1982) faisait l'hypothèse que les propriétaires-exploitants vendaient leurs terres au prix des terres en location (plutôt qu'à celui des terres vacantes) et la rente annuelle était calculée à partir des sommes perçues. Pour les exploitations de l'enquête sur la gestion des exploitations de 1977-78, ajouter la rente annuelle des terres en propriété augmentait le niveau du revenu de toutes les exploitations d'un tiers et amenait la moitié des exploitations dont le revenu ne dépassait pas un seuil arbitraire de 2000 livres au-dessus de ce seuil. Cette option est intéressante car elle prête moins le flanc aux critiques concernant son réalisme et, en outre, elle indique un moyen de libérer le patrimoine des agriculteurs par une politique agricole concrète visant à soutenir les revenus. 


\section{Discussion} et de leurs ménages doive prendre en compte leur patrimoine. Ceci concerne à la fois le niveau absolu de leurs fonds propres (car il affecte leur statut économique) et ses variations sous forme de plus et moins values en capital. Ces dernières font partie du revenu personnel mais ne sont en général pas rapportées dans les comptes de revenu collectés dans les statistiques officielles sur l'agriculture car elles ne font pas directement partie de l'activité de production. Lorsque les agriculteurs ont la capacité de choisir des manières alternatives de comptabiliser la croissance nette de leur pouvoir économique en termes de revenu courant ou de patrimoine - toute mesure partielle de leur position globale risquera de mal représenter leur situation réelle. L'importance du rapport relatif entre patrimoine et revenu dans le secteur agricole permet de transformer le revenu de manière à minimiser l'imposition et rend donc la prise en compte du capital particulièrement importante.

31 de la position des agriculteurs en matière de revenu. L'exemple du Royaume-Uni et des États-Unis montre que l'importance des plus et moins-values en capital va varier ; cellesci peuvent osciller de façon inquiétante et vont parfois à l'encontre des mouvements du revenu courant et parfois dans le même sens. Les mouvements à court terme peuvent représenter un indicateur imparfait de la tendance à plus long terme. Ces plus et moinsvalues en capital sont en grande partie hors du contrôle de l'agriculteur individuel même si les politiques agricoles et autres ont une incidence ceux elles. Si elles représentent une forme de rente économique de l'activité agricole a posteriori, les mesures du revenu courant de la production agricole - mesures conventionnelles de la prospérité des exploitations - les ignorent dans une large mesure. Il semble raisonnable de mettre en place des systèmes qui permettent de les estimer, peut-être en moyenne sur une période donnée correspondant approximativement à la durée de vie professionnelle d'une génération d'agriculteurs (disons 20 ans). de statut économique pourrait être d'importance plus grande. L'expérience tirée d'études de cas montre que ceci transforme la façon dont la pauvreté est considérée en agriculture et indique comment les décideurs de l'action publique pourraient traiter les problèmes de faibles revenus courants. La commande potentielle sur les biens et services des agriculteurs, en particulier les plus âgés, est considérablement modifiée si la rente annuelle de leurs fonds propres est également incluse. La proportion de ceux qui souffrent de pauvreté est réduite de beaucoup, même s'il restera toujours des pauvres, principalement parmi les agriculteurs non-propriétaires fonciers. Bien entendu, toute comparaison avec les autres composantes de la société, une préoccupation centrale de la politique agricole, devrait s'assurer qu'il existe des moyens d'appliquer la méthode plus complète expliquée ici pour les agriculteurs aux autres catégories socio-économiques.

Pour mettre en place un système d'information capable de produire des estimations du statut économique, on est confronté à des problèmes à la fois conceptuels et pratiques. Les informations sur le revenu courant (disponible) des exploitants agricoles et de leurs ménages au niveau microéconomique sont bonnes dans certains pays de l'OCDE mais elles sont insuffisantes dans la plupart (y compris la France et le Royaume-Uni) (Hill, 2000 ; 
OCDE, 2003). Plus sérieusement encore, les pays industrialisés semblent disposer de peu d'informations fiables sur le patrimoine de leurs agriculteurs (en particulier sur les actifs non agricoles) et sur leur comparaison avec d'autres catégories sociales. Pour développer une méthodologie d'estimation, il faudrait résoudre certaines questions théoriques centrales comme ce qui constitue un ménage agricole, mais cela ne semble pas plus difficile à faire que ce qui a été réalisé dans le domaine de la mesure du revenu du secteur des ménages agricoles (Eurostat, 2002). Resterait ensuite la question critique de la collecte des données par ménage permettant de construire les bilans. Pour les pays membres de l'Union européenne qui ont déjà des registres administratifs et la capacité d'apparier une série d'enquêtes et de banques de données, cela pourrait déjà être réalisable. Pour les autres pays, il faudrait développer de nouvelles sources de données. Comme il est probable que les estimations du statut économique ne soient pas indispensables tous les ans, il pourrait suffire d'ajouter de temps en temps des questions supplémentaires aux enquêtes. Au Royaume-Uni, l'enquête sur les exploitations agricoles (qui fait partie du RICA) est sans doute l'option la plus intéressante à court terme. Elle collecte déjà des informations partielles sur les revenus non agricoles, qui donnent indirectement des indications élémentaires sur les actifs détenus hors de l'exploitation, et qui pourraient servir de point de départ pour élargir la prise en compte des dettes. Cependant, les éventuelles difficultés de mesure ne devraient pas cacher les déficiences de l'approche actuelle et la direction dans laquelle des améliorations méthodologiques sont souhaitables.

Pour les décideurs de l'action publique, le concept de statut économique fournit des pistes pour traiter du problème des faibles revenus des ménages agricoles. Une option est de nier que les faibles revenus soient un problème lorsqu'ils sont associés à un statut économique favorable. Des estimations indicatrices du patrimoine sur la base "d'exploitations représentatives" (plutôt que des données d'enquêtes) pourraient permettre d'établir des ordres de grandeur. Les agriculteurs dont les fonds propres sont importants sont déjà en mesure de les convertir en revenu courant de diverses manières, formellement en participant à des accords de rente annuelle avec des institutions financières ou informellement en évaluant eux-mêmes leur espérance de vie, en empruntant sur la valeur des actifs et en consommant en conséquence. Cependant, les accords formels ne sont pas répandus, tout du moins au Royaume-Uni et en Amérique du Nord. Selon le RICA (Hill et Brookes, 1993), les revenus (agricoles) par personne les plus bas tendent à se trouver parmi les agriculteurs les plus âgés (à l'exception de la France) mais le niveau de l'endettement (agricole) par rapport aux actifs diminue aussi avec l'âge. Ceci suggère que le capital est peut-être important mais que les agriculteurs ne désirent pas en faire usage.

\section{Conclusion}

Les analystes des politiques pourraient discuter si cette apparente volonté de ne pas faire appel aux fonds propres est un choix délibéré ou si c'est le résultat d'une défaillance du marché qui nécessite une intervention publique. Dans les pays de l'Union européenne, il semble peu probable qu'un manque d'information, de forts coûts de transaction ou une inadéquation des institutions financières empêchent les agriculteurs d'adopter cette stratégie en cas de problèmes de niveau de revenus courants. Les agriculteurs du Royaume-Uni pourraient difficilement échapper à la publicité des banques commerciales 
encourageant les ménages âgés à participer à des dispositifs de mise à disposition du capital.

Pour les agriculteurs à faible revenu sans fonds propres importants (en fait des fermiers qui ne sont pas propriétaires fonciers), le maintien du soutien restera justifié. Qu'il transite par des instruments spécifiques à l'agriculture ou par le biais de programmes généraux de réduction de la pauvreté est une question dépassant les ambitions de cet article (même s'il devient de plus en plus difficile de justifier une approche sectorielle). Quant aux autres agriculteurs riches en actifs, il semble possible de les encourager à considérer leurs fonds propres comme une ressource permettant de résoudre les problèmes de revenu à court terme. Un cas similaire se rencontre au Royaume-Uni où les personnes âgées à faible revenu et actifs élevés doivent utiliser une partie de leurs fonds propres pour payer leurs frais de résidence en maison de retraite.

$\mathrm{Au}$ Danemark, la coutume qui fait que les agriculteurs âgés vendent leurs terres à la génération suivante (plutôt que de faire un don) pour aider à financer leur retraite représente une étape dans cette direction, mais un tel comportement est exceptionnel et ne s'applique que lorsque la terre est libérée (les méthodes de transmission des exploitations dans l'Union européenne sont passées en revue dans Perrier-Cornet et al., 1991). Pour faire plus largement accepter l'importance des fonds propres généralement élevés dans le secteur agricole, il sera nécessaire de réaliser plus largement des calculs du statut économique, de sa répartition et des comparaisons avec les autres catégories socioéconomiques. Il faut espérer que cet article encouragera de tels calculs en France et ailleurs.

\section{BIBLIOGRAPHIE}

Ahearn M., Lee J.-E. Multiple Job-holding among Farm Operator Households in the United States. In Hallberg, Findeis et Lass (Eds), "Multiple Job-holding among Farm Families”. Ames, Iowa State University Press, 1991.

Anderson F.-G., Asheim L.-J., Mittenzwei K., Veggeland F. Taxation of Agriculture in selected countries - study of The United States, Canada, Australia, Germany, United Kingdom, Ireland, France, Switzerland and Italy with relevance to the WTO. Institut norvégien de recherche économique agricole (NILF), Oslo, 2002-8.

Atkinson A.-B. Wealth, Income and Inequality. Oxford University Press, Oxford, 1980.

Bhatia K.-B. Accrued Capital Gains, Personal Income and Saving in the United States 1948-64. Review Income and Wealth, 1970, 16(4), p. 363-78.

Bhatia K.-B. On Estimating Capital Gains in U.S. Agriculture.American Journal of Agricultural Economic, 1971, 53, p. 502-6.

Bhatia K.-B. Capital Gains and the Aggregate Consumption Function. American Economic Review, 1972, 62, p. 866-79. 
Blandford D., Boisvert R. US Policy for Agricultural Adjustment. IATRC, Symposium "Adjusting to Domestic and International Agricultural Policy Reform in Industrialised Countries", Philadelphie, 6-7 juin 2004.

Brinkman G.-L. Reflections on Farm Incomes in the 1970s.Canada Journal Agricultural Economic, Actes de la réunion annuelle, 1980.

Carlin T.-A., Reinsel E.-I. Combining Income and Patrimoine: An Analysis of Farm Family Well-being. American Journal of Agricultural Economic, 1973, 55, p. 38-44.

Chase L. Inflation, Capital Gains and Farmers' Economic Well-being. Canada Journal of Agricultura Economic, Annual Meeting, 1980.

Chase L., Lerohl M.-L. On Measuring Farmers' Well-being. Canada Journal of Agricultural Economic, 1981, 29.

Eurostat. Income of the Agricultural Household Sector; 2001 Report. Eurostat, Luxembourg, 2002.

Gardner B.-L. Measuring the Income of Rural Families: Results of a Survey of Sampson County, North Carolina. North Carolina State University, Dept. of Economics, Rayleigh, 1972, Economics Research Report $n^{\circ} 20$.

Gardner B.-L. A Full Income Approach to the Measurement of Rural Poverty. North Carolina State University, Department of Economics and Business, Raleigh, 1975, Economics Research Report $\mathrm{n}$ $\circ 34$.

Hallberg M.-C., Findeis J.-L., Lass D.-A. Multiple Job-holding among Farm Families. Iowa State University Press, Ames, 1991.

Harrison A. Farmers and Farm Businesses in England. University de Reading, Department of Agricultural Economics and Management, 1975, Miscellaneous Studies 62.

Hicks J.-R. Value and Capital: An Inquiry into Some Fundamental Principles of Economic Theory. 1946, Clarendon Press, Oxford, $2^{\text {nd }}$ ed.

Hill B. Concepts and Measurement of the Incomes, Wealth and Economic Well-being of Farmers. Journal Agricultural Economic, 1982, 33(3).

Hill B. Farm Incomes, Wealth and Agricultural Policy.Ashgate Publishing, Aldershot, 2000.

Hill B., Brookes B. Farm Incomes in the European Community in the 1980s.Document series. Commission des Communautés européennes, Bruxelles, 1993.

Jégouzo G., Brangeon J.-L., Roze B. Richesse et pauvreté en agriculture. Inra, Paris, Collection économie agricole et agroalimentaire, 1998.

Melichar E. Capital gains versus current income in the farming sector. American Journal of Agricultural Economic, 1979, 61.

OCDE. Ajustement du secteur agricole des pays de l'OCDE : Réformer les politiques foncières agricoles. OCDE, Paris, 1998.

OCDE. Revenu des ménages agricoles - Questions et réponses. OCDE, Paris, 2003.

Perrier-Cornet Ph., Blanc M., Cavailhes J., Daucé p., Le Hy A. Farm Take-Over and Farm Entrance within the E.E.C., Document series, Commission des Communautés européennes, Bruxelles, 1991.

Perry G.-M., Rister E., Richardson J.-W., Leatham D.-J. The Effects of Equity Position, Credit Policy, and Capital Gains on Farm Survival. Agricultural Economics Research, 1986. 
Sexton R.-N., Duffus G.-W. On Economic Welfare and Farmer Annuity Schemes. Quarterly Review agricultural Economic, 1977, 30(2), p.117-32.

Sommer J.-E., Hoppe R.-A., Greene R.-C., Korb p.-J. Structural and Financial Characteristics of U.S. Farms, 1995: 20th Annual Family Farm Report to the Congress. Economic Research Service, US Department of Agriculture, Washington, 1998.

Vincent D.-P. Economic Aspects of Farm Poverty. Australian Journal of Agricultural Economic, 1976, 20(2), p. 103-118.

Vincent D.-P., Watson A.-S., Barton L.-M. Poverty Among Farmers in Three Districts of Victoria. Commission of Inquiry into Poverty. In Financial Aspects of Rural Poverty, Government Publishing Service, Canberra, 1975.

Weisbrod S.-A., Hansen W.-L. An income-net worth approach to measuring economic welfare. American Economic Review, 1968, 53, p. 1315-29.

Weldon R.-N., Moss C.-B., Erickson K. The Distribution of Farm Wealth in the United States. Agricultural Finance Review, 1993, 53, p. 100-9.

\section{NOTES}

1. Haern S. Farm Incomes and Capital Gains: Implications for Structural Change. PhD Thesis (unpulished), University of London, Wye College, 1977.

2. Les deux décades précédant 1972, les gains réels en capital atteignèrent en moyenne un tiers du revenu net de la ferme.

3. Voir en particulier la page 181 .

4. Tandis que les enquêtes sur le revenu des ménages considèrent souvent les membres du ménage vivant sous le même toit comme l'unité dont on mesure les revenus, il est préférable dans certains cas de ne considérer que le couple d'exploitants et ses dépendants ; c'est-à-dire que les revenus des adultes financièrement indépendants qui vivent sous le même toit ne sont pas compris, dans la mesure où ils ne font pas partie du groupe qui met ses revenus et ses dépenses en commun.

\section{RÉSUMÉS}

La situation économique des ménages agricoles est habituellement représentée par leurs revenus courants. Les plus-values sur les actifs et le patrimoine des membres du ménage ne sont généralement pas pris en compte, ce qui donne une image très partielle des ressources dont ils disposent. Le concept de «statut économique » combine à la fois le revenu et le patrimoine. Les conséquences de son utilisation dans les comparaisons entre ménages agricoles et autres catégories socio-économiques sont examinées.

The economic situation of farm households is usually described in terms of their current incomes. Capital gains on assets and the wealth owned by household members are usually ignored, thus giving a very incomplete picture of the resources at their disposal. The concept of 
"economic status" incorporates both income and wealth. The implications of using it when making comparisons of agricultural households with other groups in society are reviewed.

INDEX

Keywords : capital gains, economic status, farm households, income, wealth Code JEL D14 - Personal Finance

Mots-clés : ménages agricoles, patrimoine, plus-values en capital, revenus, statut économique

\section{AUTEURS}

\section{BERKELEY HILL}

Professeur d'analyse des politiques, Université de Londres 